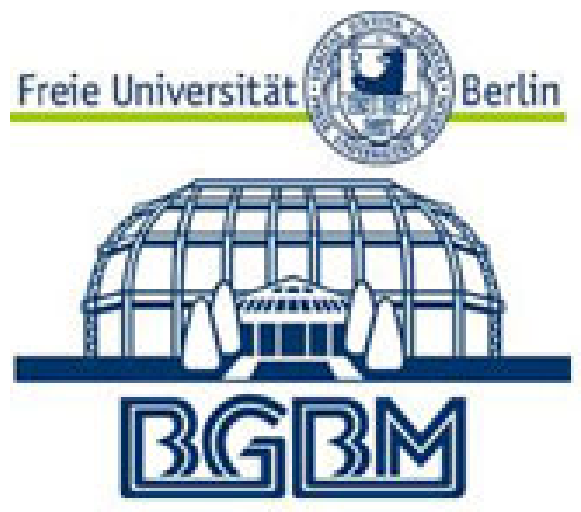

Einige neue Lupinus-Arten aus den Anden von Peru Author(s): E. Ulbrich

Source: Notizblatt des Königl. botanischen Gartens und Museums zu Berlin, Bd. 7, Nr. 69 (May 15, 1920), pp. 452-454

Published by: Botanischer Garten und Botanisches Museum, Berlin-Dahlem

Stable URL: http://www.jstor.org/stable/3994380

Accessed: 15/06/2014 05:57

Your use of the JSTOR archive indicates your acceptance of the Terms \& Conditions of Use, available at http://www.jstor.org/page/info/about/policies/terms.jsp

JSTOR is a not-for-profit service that helps scholars, researchers, and students discover, use, and build upon a wide range of content in a trusted digital archive. We use information technology and tools to increase productivity and facilitate new forms of scholarship. For more information about JSTOR, please contact support@jstor.org. 
V.

\section{Einige neue Lupinus-Arten aus den Anden von Peru.}

Von

E. Ulbrich.

1. Lupinus matucanicus Ulbrich n. sp. - Herba annua e basi ramosa caule ramisque angulosis fulvo-pilosis $10-15 \mathrm{~cm}$ altis. Foliorum stipula $\mp 15 \mathrm{~mm}$ longae subulato-lanceolatae fusco-pilosae. Folia petiolo ad $6 \mathrm{~cm}$ longo fusco-piloso instructa 9-11 foliolosa; foliola oblanceolata ad fere $20 \mathrm{~mm}$ longa 2-3 mm lata obtusa vel subacuminata supra glabra insuper margineque fuscido-pilosa. Inflorescentia terminalis sed innovationibus alaribus multo superata pedunculo fuscopiloso quam folia multo longiore; flores satis parvi ad $12 \mathrm{~mm}$ longi, coerulei, pedicello $1-1,5 \mathrm{~mm}$ longo instructi; calyx ad $7 \mathrm{~mm}$ longus, fusco-pilosus, usque fere basin partitus; vexillum retroflexum alis obliquis late-ovalibus multo brevius lamina stria alba mediana praedita; carina alis aequilonga rectangulariter curvata margine superiore subtiliter fimbriata, acuta; filamenta breviora antheris linearibus $\mp 1,5 \mathrm{~mm}$ longis; ovarium densissime fusco-setosum; fructus ignotus.

Peru: Matucana an der Lima-Oroya-Bahn; steinige Abhänge, besetzt mit einer lockeren Xerophyten-Vegetation (hauptsächlich regengrüne Sträucher), $2400 \mathrm{~m}$, abwärts bis $1800 \mathrm{~m} \mathrm{~s}$. m. (WeBerBAUER no. 5273 - fl. März 1910.)

Die Art ist verwandt mit $L$. linearis Desr., die in Brasilien vorkommt, unterscheidet sich jedoch durch breitere Blättchen und viel stärkere Beharung in allen Teilen.

2. L. pinguis Ulbrich n. sp. - Herba perennis e trunco erasso breve fasciculata, caulibus brevissimis. Foliorum stipula $\theta$ fere $2 \mathrm{~cm}$ 
longe cum petiolo connatae lobis $\mp 10 \mathrm{~mm}$ longis lanceolatis; folia petiolo crassiusculo ad $15 \mathrm{~cm}$ et ultra longo lamina 11-13-phylla foliolis uninerviis oblanceolatis ad $5 \mathrm{~cm}$ longis $10-13 \mathrm{~mm}$ latis subacuminatis utrinque pilis sericeis fulvidis ad pressis vestitis crassiusculis. Inflorescentia densissima terminalis foliis multo superata, in anthesi $\mp 6 \mathrm{~cm}$ longa pedunculo fere aequilongo valido instructa; flores pedicello $1-2 \mathrm{~mm}$ longo fulvo-sericeo instructi, calyx bilabiatus fere $7 \mathrm{~mm}$ longus dense pilis sericeis fulvidis vestitus; corolla violacea ad fere $15 \mathrm{~mm}$ longa; vexillum stria aurea mediana fere $10 \mathrm{~mm}$ longum, alae fere $12-13 \mathrm{~mm}$ longae $\mp 6 \mathrm{~mm}$ latae obliquae semiorbiculares, carina aequilonga ad fere $3 \mathrm{~mm}$ lata acuta; antherae filamentorum breviorum ovoideae; ovarium lanceolatum pilis sericeis adpressis fulvidis satis dense vestitum; stylus glaber, stigma albidopilosum capitatum. Fructus ignotus.

Peru: Hochanden zwischen $13^{0}$ und $14^{0}$ südl. Br., zwischen dem Hafen Pisco und der Gebirgsstadt Ayacucho am PaB Apacheta; Büschelgrasformation, an feuchten Stellen, 4500-4600 m.

Die schöne Art gehört in die Verwandtschaft von $L$. brevicaulis Gr. und $L$. breviscapus Ulbrich, unterscheidet sich von beiden durch die dicklichen, etwas fleischigen Blätter und die bräunlichgelbe, seidige Behaarung. Von L. brevicaulis Gr. ist sie verschieden durch kürzere und breitere Blätter und kürzeren Blütenstand.

3. L. misticola Ulbrich n. sp. - Herba annua e basi pauceramosa caule erecto vel ascendente pilis fulvidis primum vestito postea glabrescente. Foliorum stipula $\theta$ cum petiolo fere $2 \mathrm{~cm}$ longe connatae lobis ad fere $8 \mathrm{~mm}$ longis fulvo-sericeis anguste-lanceolatis. Folia petiolo ad fere $20 \mathrm{~cm}$ longo rimuloso glabrescente instructa lamina $\mp 9$ phylla, foliola lanceolata ad fere $5 \mathrm{~cm}$ longa, $8-10 \mathrm{~mm}$ lata, acuta crassiuscula supra glabra, infra parcius pilis fulvidis sericeis vestita, uninervia. Inflorescentia terminalis pedunculo ad $40 \mathrm{~cm}$ et ultra alto pilis sericeis caducis dispersis vestito instructa, folia longe superans; bracteae ex ovato lanceolatae ad fere $8 \mathrm{~mm}$ longae longicuspes pilosae; pedicelli ad fere $10 \mathrm{~mm}$ longi fulvido-pilosi, calyx oblique campanulatus bilabiatus fere $8 \mathrm{~mm}$ longus fuscido-pilosus, corolla coerulea vexillum retroflexum secundum medium aureum, alae late ovatae obtusae fere $12 \mathrm{~mm}$ longae $7 \mathrm{~mm}$ latae; carina aequilonga fere $5 \mathrm{~mm}$ lata apice atro-coerulea; antherae breviores lineares fere 1,5 mm longae, ovarium densissime pilosum, stylus glaber, stigma subdiscoideum albo-pilosum. Fructus immaturus fere $3 \mathrm{~cm}$ longus $1 \mathrm{~cm}$ latus, 3-4-spermus, densissime pilis sericeis fulvidis vestitus. Semina sublentiformia $3 \times 4 \mathrm{~mm}$ lata et longa, alba. 
Peru: Ostseite des Vulcans Misti bei Arequipa, $3800 \mathrm{~m} \mathrm{s.} \mathrm{m.}$ auf sandigem, dürftig bewachsenem Boden zwischen Büschelgräsern und Tolasträuchern. (A. Weberbauer no. 1423 - fl. et fr. 8. September 1902.)

Die Art gehört in die Verwandtschaft von Lupinus panniculatus Desr., unterscheidet sich jedoch durch den viel höheren und reicheren Blütenstand, die größeren, breiteren und viel stärker behaarten Früchte und die langgestielten, kahleren, spitzeren und größeren Blätter. 